\title{
Hypertension and microalbuminuria in HIV infected patients: beneficial effects of the treatment with telmisartan
}

\author{
Claudio Ucciferri, Paola Mancino, Katia Falasca, Francesca Vignale, Jacopo Vecchiet \\ From $16^{\text {th }}$ International Symposium on HIV and Emerging Infectious Diseases \\ Marseille, France. 24-26 March 2010
}

\section{Background}

In HIV infected patients there is increasing evidence of hypertension and microalbuminuria, two important risk factors for renal and cardiovascular disease (CVD). Antihypertensive drugs inhibiting the renin-angiotensin system exert an antiproteinuric effect. Telmisartan, an angiotensin II receptor blocker partial agonist of the PPAR- $\gamma$ approved for the treatment of hypertension, seems to exert a nephro-protective effect independent of blood pressure reduction in the general population. Aim of the study was to evaluate kidney-protective effects of telmisartan in hypertensive HIV+patients with microalbuminuria.

\section{Methods}

8 Caucasian male HIV+ patients cART treated without therapeutic changes for over 12 months and recent diagnosis of mild hypertension, were treated with telmisar$\tan 80 \mathrm{mg}$ daily. They had suppressed viremia and CD4 cell count $>300 \mathrm{cell} / \mathrm{ml}$ up on 6 month, and microalbuminuria $>5 \mathrm{mg} / \mathrm{l}$. Systolic (SBP) and diastolic (DBP) blood pressure, triglycerides (TGs), total cholesterol (TCh), HDL (HDL-C) and LDL (LDL-C) cholesterol, CRP, ESR, microalbuminuria, MDRD-GFR, cystatin-C, IL-18, VEGF and endothelin-1 were measured at baseline (T0), one (T1), three (T3) and six months (T6). All the statistical analysis was performed with the SPSS Advanced Statistical 7.5 Software.

\section{Results}

Treatment with telmisartan improved SBP and DBP values at $\mathrm{T} 1$ yet $(\mathrm{p}=0,001)$. Microalbuminuria were statistically decreased at $\mathrm{T} 1(\mathrm{p}=0,006)$ and further on T6 ( $\mathrm{p}=0,0001)$, whereas MDRD-GFR was statistically augmented $(\mathrm{p}=0,03)$. Cystatin- $C$, endothelin-1 and VEGF were statistically reduced at T3 $(\mathrm{p}=0,0001 ; \mathrm{p}=$ 0,01 and $\mathrm{p}=0,0045$ respectively) and at T6. TG, TCh, LDL-C levels decreased with statistical significance at T6 ( $\mathrm{p}=0,003 ; \mathrm{p}=0,03$ and $\mathrm{p}=0,02$ respectively), while HDL-C increased at T6 $(\mathrm{p}=0,04)$. ESR, PCR and IL-18 decreased at T6 ( $\mathrm{p}=0,04 ; \mathrm{p}=0,006$ and $\mathrm{p}=0,02$ respectively).

\section{Discussion}

Telmisartan was well tolerated and effective to improve hypertension and lipid metabolism. Decreased microalbuminuria and cystatin-C with increased MDRD-GFR are indicative of nephro-protective effects of telmisartan. Mechanisms causing microalbuminuria in HIV+ patients could be related to infection, chronic inflammation and endothelial dysfunction. Decreased endothelin-1 and VEGF in this study may be related to an endothelial protective effect of telmisartan. These data confirm renal and endothelial protective effects of telmisartan also in HIV+ patients.

Published: 11 May 2010 